\title{
BRIBE AND PUNISHMENT: EFFECTS OF SIGNALING, GOSSIPING, AND BRIBERY IN PUBLIC GOODS GAMES
}

\author{
A. L. C. BAZZAN* and SÍLVIO R. DAHMEN ${ }^{\dagger}$ \\ *Inst. de Informática, UFRGS, C.P. 15064 \\ 91501-970 P. Alegre, RS, Brazil \\ †Inst. de Física, UFRGS, C. P. 15051 \\ 91501-970 P. Alegre, RS, Brazil \\ *bazzan@inf.ufrgs.br \\ †dahmen@if.ufrgs.br
}

Received 10 February 2009

Revised 15 August 2010

\begin{abstract}
In public goods games, individuals contribute to create a benefit for a group. However this attracts free-riders, who enjoy the benefits without necessarily contributing. Nonetheless, in real-life scenarios cooperation does not collapse. Several explanations have been proposed in order to explain this phenomenon, such as punishment and signaling. In the present work, we investigate the effects of new elements associated with punishment upon signaling such as gossiping and bribery. Agents may denounce freeriders (who on their turn get punished) or may be bribed to remain silent and even spread rumors of false good behavior. Having a model with richer social mechanisms enable us to test how cooperation develops in situations in which players have social attachments. Our results show that when punishment and bribery are present, the levels of contribution are kept at a relatively higher value compared to the situation when no punishment is exercised. As to what regards gossiping, if the number of free-riders is high, finding mechanisms to prevent gossiping could be an important step in order to increase the contribution. If the ratio between free-riders and other agents is about one, then gossiping does affect contribution in a positive way.
\end{abstract}

Keywords: Public goods game; cooperation; agent-based simulation.

\section{Introduction}

The evolution of cooperation has been extensively studied by biologists, whose theoretical results have motivated both game theoreticians as well as computer scientists. Just to name one important issue under investigation, which is relevant for social and economic systems, there is the question of why people do cooperate in situations in which one would expect rational decision-making to lead to no cooperation. Moreover, one observes cooperation at many levels of biological organizations. Evolution of cooperation has many aspects (see e.g. [14] and references therein where some important mechanisms for the evolution of cooperation are discussed, not only among humans but also among other species). 
In this paper, we address two main mechanisms to investigate cooperation: sanctioning (punishment), and the so called "green beard" model where cooperators recognize each other via arbitrary labels. These two mechanisms seem to play an important role in cooperation among humans, especially in modern societies. One can think about the consequences of bad reputation and loss of cooperation among partners in electronic commerce: the internet allows not only networking and long lasting relationship among web users but also provides the basis to increase the collective memory about who is who in cyberspace. This in turn helps create mechanisms to distinguish and punish non-reciprocators. Although it is not a consensus that punishment is a mechanism for the evolution of cooperation, it is an important factor that can promote cooperative behavior in some situations [14]. In fact Gürek et al. [11] report laboratory experiments with real (human) subjects playing the public goods game when two subjects can select among a sanctioning and a sanctioning free institution. Their results show that subjects do migrate to the sanctioning one and cooperate, thus demonstrating the competitive advantage of sanctioning.

Besides this sanction mechanism, we also explore, in a loose sense, the so-called green-beard model, whose underlying idea is due to Hamilton [12] while the term itself was coined by Dawkins [5]. In the latter, the author makes the point about a hypothetical green-beard effect, i.e. a metaphor for a physical trait. Dawkins suggests that if a gene arises that not only gives individuals a very distinguishable physical trait but also a tendency to be altruistic towards other individuals which depict the same trait, mutual altruism between individuals having that trait could evolve. Despite Dawkins own speculation about the unlikelihood of the existence of a gene that would produce such a trait and altruistic tendencies, evidences were found in 1998 that a green-beard gene exists in the fire ant, Solenopsis invicta.

In the present paper, we use the green-beard effect as a metaphor to designate individuals that are genetically programmed to behave altruistically towards individuals that have green beards (similar tags) in the context of public goods games. Games in phenotype space are investigated also by Nowak et al. [16] but they use other games (e.g. prisoner's dilemma) to analyze evolution of cooperation. Also using this game, Antal et al. [1] show that cooperation is favored when phenotype mutation rate is large. Here, individuals only interact with others with same phenotype.

The objective is to investigate the role played by mechanisms such as punishment and signaling in the evolution of cooperation among synthetic persons in a public goods game by means of agent-based simulation. These two main mechanisms can be seen as proxies for more complex issues such as emotions, sense of fairness, as well as mechanisms such as gossiping and bribery, which appear in our model.

Therefore, the contribution of this paper is the introduction of new elements in the study of the public goods game, trying to bring the model closer to real world situations. We start by analyzing the behavior of agents when they interact in a grid, can punish, are subject to bribery, and can spread gossips about their acquaintances. 
In public goods games, individuals incur a cost to create a benefit for a group. They are social dilemmas because free-riders do enjoy the benefits created by the group without contributing themselves. Since free-riders are attracted by the benefits and may proliferate, one may expect that eventually cooperation will collapse. However, human societies have somehow managed to solve this. Therefore, there has been a great interest in public goods problems or dilemmas; many researchers try to contribute to an understanding of the nature of these problems. The most popular explanations are based on signaling, reputation, and sanctions. See [13] for an overview.

In our model of the public goods game, the green beard effect means that individuals contribute when they see other green beards. We also use the notion of avoiding contribution if the individual does not see such beards. Thus, the idea of green beards is here modified to include two kinds of beards: the blue and the red ones. These colors come from the fact that they were used to depict cooperation and defection respectively in the seminal work of Nowak and May [15] regarding evolution of cooperation in spatial IPD. Thus we keep those colors to indicate cooperation and free-riding also in the public goods game.

These mechanisms for the investigation of the dynamics of cooperation in public goods games are formulated in details in Sec. 3. In the next section, we discuss other works related to the public goods game. Section 4 then presents and discusses the scenario and details of the simulation settings as well as the results. In Sec. 5, we make our concluding remarks.

\section{Public Goods Game}

In its original formulation, this game deals with public spending on community roads, libraries, etc. Players in the game are given the opportunity to contribute to a common pool. Benefits (obtained from tolls, membership fees) are equally distributed among all participants irrespective of their contributions. Clearly it would be "fair" for people to pay the same quantity for those items. However individuals are different, as they have different social and economic conditions and different stances which means that some contribute less than others. This being common-knowledge, if one assumes each player as rational s/he would default and contribute nothing. However this is not what occurs in reality.

In the literature, it has been shown that altruistic behavior can prevail either if players use imitation and their interactions are local (e.g. [3, 2, 8]), or in the context of strong reciprocity [9]. A strong reciprocator is predisposed to cooperate with others and punish non-cooperators, even when this behavior cannot be justified in terms of extended kinship or reciprocal altruism.

Regarding the public goods game in particular, there has been several interesting research directions. Gintis et al. [10] formulate an explanation of cooperation among unrelated members of a social group in which cooperation evolves because it constitutes an honest signal of the member's quality as a mate, coalition partner or competitor, and therefore results in advantageous alliances for those signaling in 
this manner. To draw this conclusion, the authors use a public goods game scenario that involves no repeated interaction. They show that honest signaling of underlying quality by providing a public good to group members can be evolutionarily stable provided that certain plausible conditions hold. However their setting does not involve the dynamics of repeated games, nor issues such as spread of knowledge about tags of other players.

Gürek et al. [11] have run laboratory experiments with real subjects playing this game when two subjects can select among two institutions to contribute: a sanctioning and a sanction-free one. The usual argument against punishing is that it is associated with a cost: a rational agent should refrain from punishing in order to avoid extra costs. However, recently Bowles and Gintis [4] have used an agent-based model of cooperation and punishment to show that the behavior of strong reciprocators, which are individuals willing to reward fair behavior and punish unfair ones even if they gain nothing with it, can be evolutionary stable.

Besides experimental results, there has been also studies on the public goods game which rely on simulation and/or analytical formulation. In [19], the evolution of dynamics of the relationship among agents that interact with the two closest neighbors is described. The contribution made by agents depends on how the closest neighbors contribute. This was later extended in [18] where the authors have studied the changes in persistence when agents are no longer locally constrained; rather, they interact in a small-world scenario.

Santos et al. [17] recently showed that cooperation is enhanced when social ties follow a scale-free distribution and all individuals contribute a fixed amount irrespective of the number of public goods games in which they engage. However it seems that in order to achieve a high level of cooperation, the so-called enhancement factor $(\eta)$ must be high.

In [7], de Jong et al. show that it is possible to represent fairness in multiagent interactions. They have implemented a descriptive model of human fairness (Homo egualis) that is used in an adaptive multi-agent system. The focus of the paper is on the development of an architecture to allow the inclusion of such a descriptive model of fairness, although experiments with the ultimatum game were performed showing that valid solutions of the game were found. This was later extended [6], resulting in a methodology that allows agents to learn and maintain cooperation in games of continuous space of strategies such as the public goods game.

\section{Modeling}

The aim in our experiments with an artificial population of agents is to investigate how real people make decisions in public goods games. One theory is that the decision is not purely rational. Rather, people have an idea of fairness that is reflected in the interactions. It is not easy to model this notion of fairness, especially because 
Table 1. Parameters used in the model with social mechanisms.

\begin{tabular}{ll}
\hline Parameter & \\
\hline$n$ & Total number of agents \\
$\mathcal{N}_{i}$ & List of neighbors of $i$ \\
$\mathcal{B}_{i}$ & List of agents believed to have blue tags \\
$\mathcal{R}_{i}$ & List of agents believed to have red tags \\
$p_{R}$ & Percentage of free-riders (red beard) \\
$r$ & Interest rate \\
$c_{m e}$ & Cost to punish \\
$c_{y o u}$ & Cost if punished \\
$\omega$ & Willingness to punish \\
$p_{g}$ & Probability of propagating gossip \\
$\Sigma_{t}$ & Number times played so far \\
$t_{\max }$ & Number of steps to play \\
$q_{\min }$ & Minimum contribution \\
$q_{\max }$ & Maximum contribution \\
$q_{\min } C$ & Minimum contribution of type HC \\
$q_{i}$ & Contribution of $i$ \\
$\left.\bar{q}_{(} t\right)$ & Average contribution over all individuals \\
$R_{i}(t)$ & Return obtained by player $i$ at $t$ \\
$W_{i}$ & Accumulated wealth of $i$ \\
$w_{0}$ & Initial quantity of money \\
$\bar{w}_{i}$ & Average wealth of $i$ over time \\
$\beta_{i}$ & Bribe offered by $i$ (as percentage of $\bar{w}_{i}$ ) \\
$\lambda_{i}$ & Factor applied to $w_{i}$ to compute minimum bribe accepted by \\
\hline &
\end{tabular}

it changes from individual to individual. Thus agent-based simulation is a powerful tool to carry out that investigation.

In what follows, we describe our model. The major parameters used are described in Table 1; these appear in nearly all models reported in the literature. When possible and appropriate, the model is simulated here with the same parameters' values.

Before we discuss the particularities of our model, we briefly explain the basic behavior that is commonly found in the literature.

At each timestep $t<t_{\max }$, each individual $i \in 1, \ldots, n$ selects a value $q_{i}(t) \in$ $\left[q_{\min }, q_{\max }\right]$ to contribute. The overall contribution is multiplied by $r$. This can be seen as a factor by which some interest provider (e.g. a bank) increases the overall wealth. The average contribution over all individuals is computed as in Eq. (1). Then, the return per agent is as in Eq. (2).

$$
\begin{aligned}
\bar{q}(t) & =\frac{1}{n} \sum_{i=1}^{n} q_{i}(t), \\
R_{i}(t) & =\bar{q}(t) \times r-q_{i}(t) .
\end{aligned}
$$

At each timestep, each agent has an accumulated wealth given by Eq. (3), where at time $t=0$, the initial wealth of each agent is $w_{0}$.

$$
W_{i}(t+1)=W_{i}(t)+R_{i}(t) .
$$


Given this general description of the public goods game, we now discuss the specificities of our model that aims at investigating the role played by mechanisms such as signaling, punishment, bribery, and gossiping. We follow other works and let agents interact in a grid. Remember that signaling is implemented by means of agents recognizing contributors (blue beards) and free-riders (red beards). This behavior varies from agent to agent. Some may perform very simple associations regarding reputation such as "blue beards are contributors" (among others) and behave accordingly; others may be prone to punish upon recognizing free-riders; some others may be bribed not to punish in such situations. Also, agents can gossip about their acquaintance, spreading rumors about them, bribers included.

Furthermore, agents can observe how much their close neighbors have contributed, and may then punish bad behavior. Those who punish spend $c_{m e}$, but those punished have to pay $c_{y o u}$.

In summary, the decision has three main steps:

(1) decide how much to contribute $\left(q \in\left[q_{\min }, q_{\max }\right]\right)$;

(2) observe neighbors; update list of blue beards $\left(\mathcal{B}_{i}\right)$ and red beards $\left(\mathcal{R}_{i}\right)$; decide whether and how much to punish; opportunity to bribe;

(3) propagate gossips about other players.

The basis of the behavior of each agent appears in Algorithm 1. Initially, agents are created with tags: red for free-riders (FR) and blue for high-contributors (HC). Free-riders contribute $q_{\min }=0$. Contributors donate a quantity between $q_{\min _{H C}}$ and $q_{\text {max }}$. For these, the exact quantity is defined by the knowledge each contributor has about other agents' tags. Each contributor $i$ compares $\mathcal{B}_{i}$ and $\mathcal{R}_{i}$ in order to decide how much to contribute. It contributes $q_{\max }$ if it sees more high-contributors than free-riders (line 12 in Algorithm 1). Conversely, if it sees more free-riders than high-contributors, it contributes $q_{\min _{H C}}$ (line 10). In other cases it contributes a quantity randomly selected between $q_{\min _{H C}}$ and $q_{\max }$. The total contributed is then multiplied by $r$ and divided equally among the $n$ participants (line 18). The accumulated wealth $W_{i}$ involves not only the return $R_{i}$, but also $q_{\max }$ and the amount actually contributed $\left(q_{i}\right)$ from which the average wealth of $i$ over time $\left(\bar{w}_{i}\right)$ is computed (line 20).

The next step is to decide whether or not to punish. Any member of the $\mathcal{R}_{i}$ list can be punished. Notice that because of gossiping (see below), this list may grow with time and may hence contain not only neighbors. In order to punish, agents look at the internal threshold (to punish). They will only punish if their average wealth $\left(\overline{w_{i}}\right)$ is higher than the cost of punishing $\left(c_{m e}\right)$ multiplied by the factor $\omega$. If this is the case, then it will punish somebody drawn randomly from its $\mathcal{R}_{i}$ list (line 24). Those punished have may have their balance decreased by $c_{\text {you }}$ while those who had punished may lose $c_{m e}$. Before this actually happens however, the punisher can be bribed by the agent to be punished, if they are neighbors (we assume that bribery can only happen in close neighborhood). 


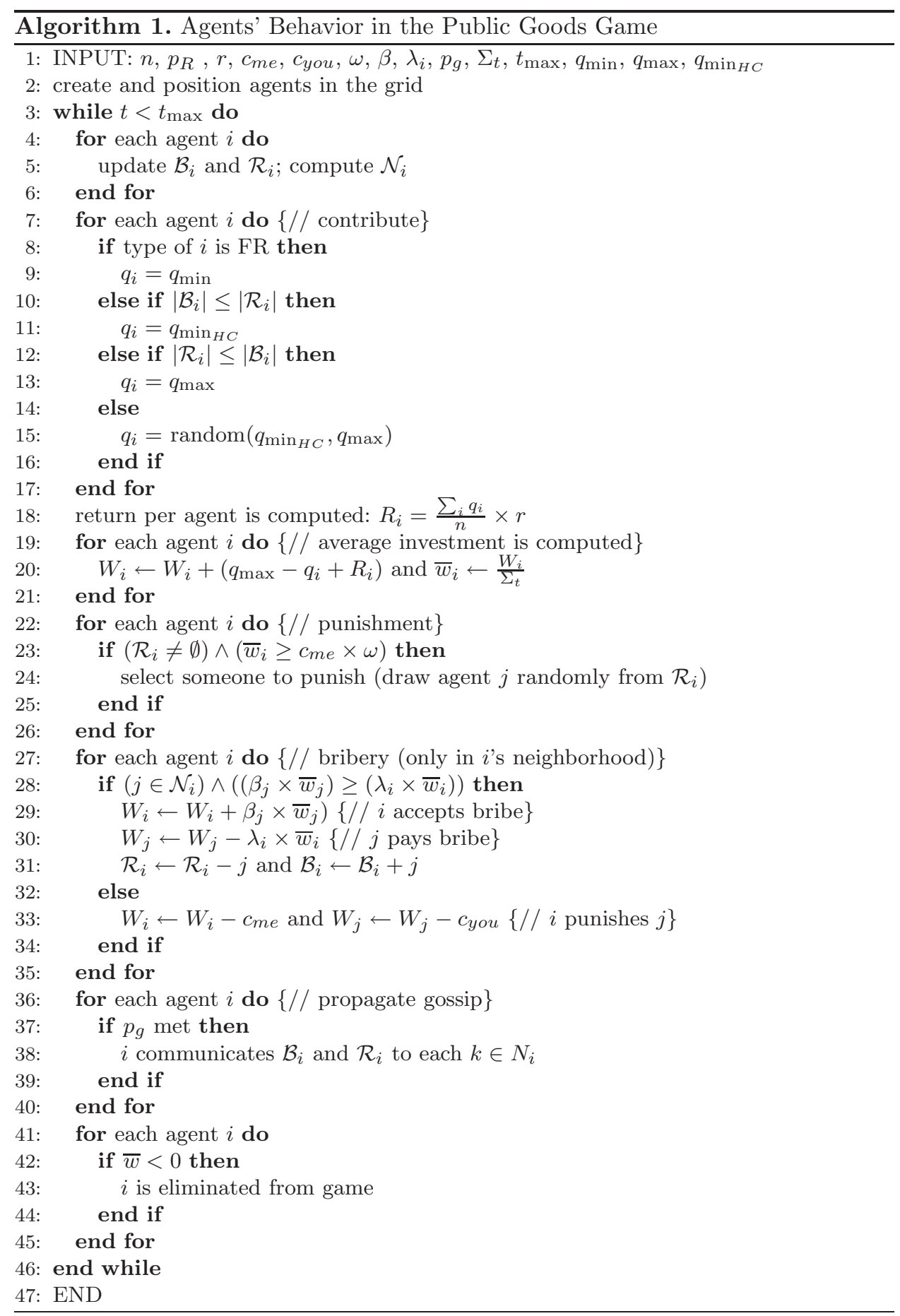


For a bribe to be accepted, its value must be superior to the internal threshold each agent has. We set this threshold the following way: agent $i$, when created, is associated with a factor $\lambda_{i}$ whose value is drawn from a normal curve with mean 2 and deviation $1 . \lambda_{i}$ is multiplied by $\overline{w_{i}}$ at each round meaning that if $i$ is to be bribed then the bribe must be superior to $\lambda_{i} \times \overline{w_{i}}$. Of course this information is not public. Similarly, a briber cannot pay more than its average wealth $\overline{w_{i}}$. Thus each potential briber has also a threshold to determine how much to offer. When $j$ is created, it is associated with a factor $\beta_{j}$ which is a number from 0 to 1 . Thus each time $j$ must bribe somebody, it will offer $\beta_{j} \times \overline{w_{j}}$. Thus, when an agent $i$ wants to punish another agent $j, j$ will offer a bribe and if this is superior to what $i$ sets as its minimum bribe, the deal happens, meaning that not only $i$ will not apply the punishment to $j$, but also that $i$ will delete $j$ from its $\mathcal{R}_{i}$ list and add it to its $\mathcal{B}_{i}$ list. $j$ transfers the bribe to $i$ and the accumulated wealth of both are updated (lines 29 and 30).

In the last stage, agents may spread gossips to their neighbors. Each agent has a probability $p_{g}$ of doing this at each time step. When this is fulfilled, $i$ sends a message to each $k$ in its neighborhood. This message contains lists $\mathcal{R}_{i}$ and $\mathcal{B}_{i}$. Therefore, a gossip will be spread about red and blue beards which may not be true given that bribery may have happened. Such lists will propagate further in the grid. Each time an agent receives a message, it checks against its own neighbors. This prevents an agent $i$ believing in a gossip about its own neighbor. Given the close relationship, it is reasonable to assume that $i$ can check the real beard color of any $k$ th neighbor. At the end of each round, agents with negative average wealth are eliminated.

It is important to notice that any agent can punish, bribe, and gossip; even a free rider. After all it may punish on its own interest, as the less people contribute, the less it will receive. Thus free-riders also have interest in eliminating other freeriders. Because they normally perform well (unless heavily punished), they may afford punishment.

\section{Simulations and Results}

This section presents and discusses experiments using the model just introduced. As shown next, the signaling mechanism (beard color) has an effect on the contribution: the more red beards are seen, the lesser the contribution, which reflect the common sense. The insertion of gossiping, already at low rates, has the effect of disseminating information throughout the grid. The increase in information potentially leads to more punishment, even if sometimes followed by bribery, thus decreasing wealth. However it may cause free-riders to go bankrupt, thus eventually allowing an increase in the rate of contribution.

In order to see this, in the following experiments, values for the parameters are as follows: the grid size is $15 \times 15$ hence $n=225$. Furthermore, $r=1.2 ; c_{m e}=5$; $c_{\text {you }}=20 ; \omega=3 ; q_{\min }=0 ; q_{\min _{H C}}=10 ;$ and $q_{\max }=20$. 
Table 2. Analytical values of $\left\langle\overline{w_{i}}\right\rangle$ and $\left\langle q_{i}\right\rangle$, for different percentages of free-riders.

\begin{tabular}{clc}
\hline$p_{R}(\%)$ & $\left\langle\overline{w_{i}}\right\rangle$ & $\left\langle q_{i}\right\rangle$ \\
\hline 25 & 22.25 & 11.25 \\
50 & 21.5 & 7.5 \\
75 & 20.75 & 3.75 \\
\hline
\end{tabular}

In order to analyze the results of the simulations, we plot the global average wealth and the global average contribution (henceforth $\left\langle\overline{w_{i}}\right\rangle$ and $\left\langle q_{i}\right\rangle$ respectively). These are averaged over all agents' $\overline{w_{i}}$ (average wealth) and over all agents' $q_{i}$ (individual contribution) respectively. We have run repetitions of each type of simulation 30 times and show the respective standard deviations.

We do not show all plots. Rather, we plot one typical case (Fig. 1) as well as the cases that deviate from it. By typical here, we mean that curves for $\left\langle\overline{w_{i}}\right\rangle$ and $\left\langle q_{i}\right\rangle$ have the same pattern (convergence to a stable level) even if they differ quantitatively (converging to a different value). Typical cases are then summarized in Table 3 where we present these levels at the end of the simulation time.

Within any given simulation, there is of course a strong variation in the performance of individual agents. This happens first because they have different types (free-riders, high-contributors), different willingness to punish, different information, and different thresholds to bribe or be bribed. We briefly discuss some individual cases at the end of this section.

Experiments were run changing the percentage of free-riders in the population of $n$ agents, as well as: (i) including punishment and bribery (or not); (ii) including

Table 3. Average wealth and average contribution at the end of the simulations. $p_{R}$ is the rate of free-riders; $p_{g}$ is the rate of gossip; averages and deviations are over 30 runs.

\begin{tabular}{|c|c|c|c|c|c|c|c|}
\hline \multicolumn{3}{|c|}{ Type of simulation } & \multicolumn{2}{|c|}{$\left\langle\overline{w_{i}}\right\rangle$} & \multicolumn{2}{|c|}{$\left\langle q_{i}\right\rangle$} & \multirow[b]{2}{*}{ Remark } \\
\hline$p_{R}(\%)$ & punish. & $p_{g}(\%)$ & avg. & std. & avg. & std. & \\
\hline 25 & no & 0 & 22.8 & 0.0 & 13.9 & 0.2 & - \\
\hline 25 & yes & 0 & 22.7 & 0.4 & 19.3 & 0.3 & - \\
\hline 25 & yes & 20 & 23.7 & 0.1 & 19.9 & 0.1 & see Fig. 1 \\
\hline 25 & yes & 50 & 23.8 & 0.0 & 20.0 & 0.0 & - \\
\hline 25 & yes & 70 & 23.8 & 0.0 & 20.0 & 0.0 & - \\
\hline 50 & no & 0 & 21.5 & 0.0 & 7.7 & 0.2 & - \\
\hline 50 & yes & 0 & 16.9 & 0.3 & 12.9 & 0.6 & - \\
\hline 50 & yes & 20 & 20.2 & 1.9 & 19.2 & 1.4 & see Fig. 2 \\
\hline 50 & yes & 50 & 19.9 & 3.5 & 18.4 & 2.5 & see Fig. 3 \\
\hline 50 & yes & 70 & 17.8 & 3.9 & 16.8 & 3.0 & see Fig. 4 \\
\hline 75 & no & 0 & 20.6 & 0.0 & 3.0 & 0.1 & - \\
\hline 75 & yes & 0 & 16.8 & 0.7 & 7.4 & 0.7 & - \\
\hline 75 & yes & 20 & 14.6 & 0.3 & 8.3 & 0.8 & - \\
\hline 75 & yes & 50 & 14.3 & 0.3 & 8.0 & 0.9 & - \\
\hline 75 & yes & 70 & 14.3 & 0.2 & 8.2 & 1.0 & - \\
\hline
\end{tabular}


gossiping (or not); (iii) changing the probability of gossip spreading. These settings can be seen in Table 3 (first 3 columns).

Before discussing the simulation results, we show simple calculations of expected values considering the percentage of free-riders $\left(p_{R}\right)$ only (i.e. without considering any locality or information factor, nor cost of punishing and bribery, nor gossiping) for later comparison. The average expected contribution $\left\langle q_{i}\right\rangle$ is given by Eq. (4) where $\overline{q_{i}}=\frac{q_{\max }+q_{\min }{ }_{H C}}{2}$.

Similarly, the average expected wealth can be computed as in Eq. (5).

$$
\begin{gathered}
\left\langle q_{i}\right\rangle=p_{R} \times q_{\min }+\left(1-p_{R}\right) \times \overline{q_{i}} \\
\left\langle\overline{w_{i}}\right\rangle=p_{R} \times\left(q_{\max }+\left\langle q_{i}\right\rangle \times r\right)+\left(1-p_{R}\right) \times\left(q_{\max }-\overline{q_{i}}+\left\langle q_{i}\right\rangle \times r\right) .
\end{gathered}
$$

Thus, for $p_{R}=25 \%, p_{R}=50 \%$, and $p_{R}=75 \%$, we have $\left\langle q_{i}\right\rangle$ and $\left\langle\overline{w_{i}}\right\rangle$ as in Table 2.

These values can now be used to evaluate the effect of the additional elements introduced in the game. We first analyze the case without punishment (and bribery), and with no gossiping (first line of each group in Table 3).

Global average wealth is of course an important measure but because punishment is associated with costs $\left(c_{m e}\right.$ and $\left.c_{y o u}\right)$, it is not fair to compare cases with and without punishment in a straight way, i.e. these costs would have to be taken into account. Therefore, the most important measure is the actual global average contribution $\left\langle q_{i}\right\rangle$ because it shows how much agents have actually contributed in each of the simulated cases.

Comparing the expected values (Table 2) with the actual ones (Table 3), we can see that the actual contribution $\left\langle q_{i}\right\rangle$ is significantly higher than expected when few free-riders exist. This is an effect of the local information agents can collect in their neighborhood. The fact that their blue beard lists are probably higher than their red beard lists, leads high-contributors to an actual contribution that is higher than simply $\frac{q_{\max }+q_{\min }{ }_{H C}}{2}$. As expected, when the rate of free-riders increase, the opposite happens, namely that the actual contribution is nearly the same or lower than the expected one.

What happens when punishment (and hence bribery) but no gossiping can be exercised? Then some agents bear the cost of punishment and the whole population bear the cost of those punished. Therefore it is expected that wealth will be reduced. This is the case as seen for instance when $p_{R}=50 \%\left(\left\langle\overline{w_{i}}\right\rangle=17\right)$. We remark however that when $p_{R}=25 \%$, the overall wealth $\left\langle\overline{w_{i}}\right\rangle$ remains at the same level because not so many agents must be punished. It also must be noticed that $\left\langle\overline{w_{i}}\right\rangle$ is computed over a cumulative quantity (see line 21 of Algorithm 1 for the computation of $W_{i}$ ). Thus there is an inherent inertia in this quantity.

If we look at the overall average contribution $\left\langle q_{i}\right\rangle$, which is instantaneous, we notice an increase in contribution no matter the initial percentage of free-riders: the level of contribution is higher when local beard colors can be observed (as 
compared with the expected value, which is lower than when punishment is exercised). This happens because information and punishment cause agents to punish free-riders that are eventually eliminated.

Now we discuss in more detail what changes when gossiping is added. We have tested three rates of dissemination of gossip $\left(p_{g}\right): 20,50$ and $70 \%$ (besides the zero rate already discussed) for each rate of free-riders. For $p_{R}=25 \%$ wealth $\left\langle\overline{w_{i}}\right\rangle$ increases slightly to 24 (as compared to 23 when no gossip is allowed). Again there is inertia in this value.

Contribution $\left\langle q_{i}\right\rangle$ increases to 20 , thus much higher than the expected value of 11.25 and also higher than those cases with local information only (14), and with punishment and bribery (19). There is not much difference in $\left\langle q_{i}\right\rangle$ when $p_{g}$ varies, probably because the blue beard lists are bigger than the red ones anyway.

As to what regards the asymptotic behavior of the game, we observe a symmetry high-contributors $\leftrightarrow$ free-riders in the sense that when one or the other dominates, the shape of the curves are similar. One has a qualitatively equal curve, whose limiting values are however different, as in one case more agents contribute and in the other case less do so. One case of such behavior namely for $p_{R}=25 \%$ and $p_{g}=20 \%$ is depicted in Fig. 1. The other cases are similar; asymptotic behaviors at the end of the simulations appear in Table 3.

On the other hand, when the number of free-riders and contributors is equal, a richer dynamics arises. It takes longer for $\left\langle\overline{w_{i}}\right\rangle$ to reach levels observed in the experiments with $25 \%$ and $75 \%$ of red beards. Associated with this, note that deviations are higher both for $\left\langle\overline{w_{i}}\right\rangle$ and $\left\langle q_{i}\right\rangle$. The inflexion in the curves regarding $\left\langle\overline{w_{i}}\right\rangle$ when $p_{R}=50 \%$ may be due to the high rate of punishment which reduces

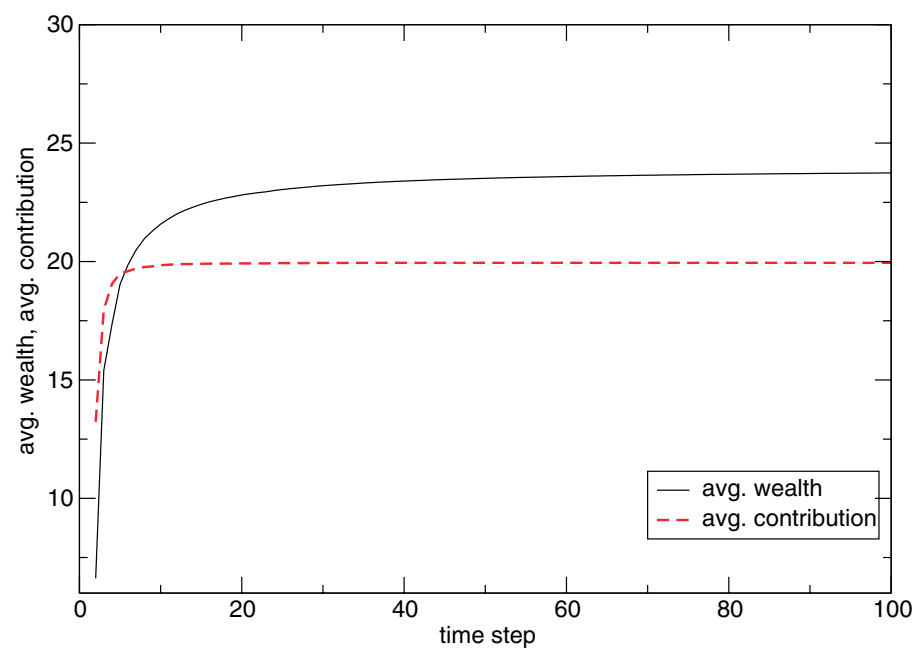

Fig. 1. Average wealth $\left(\left\langle\overline{w_{i}}\right\rangle\right)$ and contribution $\left(\left\langle q_{i}\right\rangle\right)$ as a function of time (steps); for low rate of free-riders $\left(p_{R}=25 \%\right)$; with punishment plus bribery, and low rate of gossiping $\left(p_{g}=20 \%\right)$. 


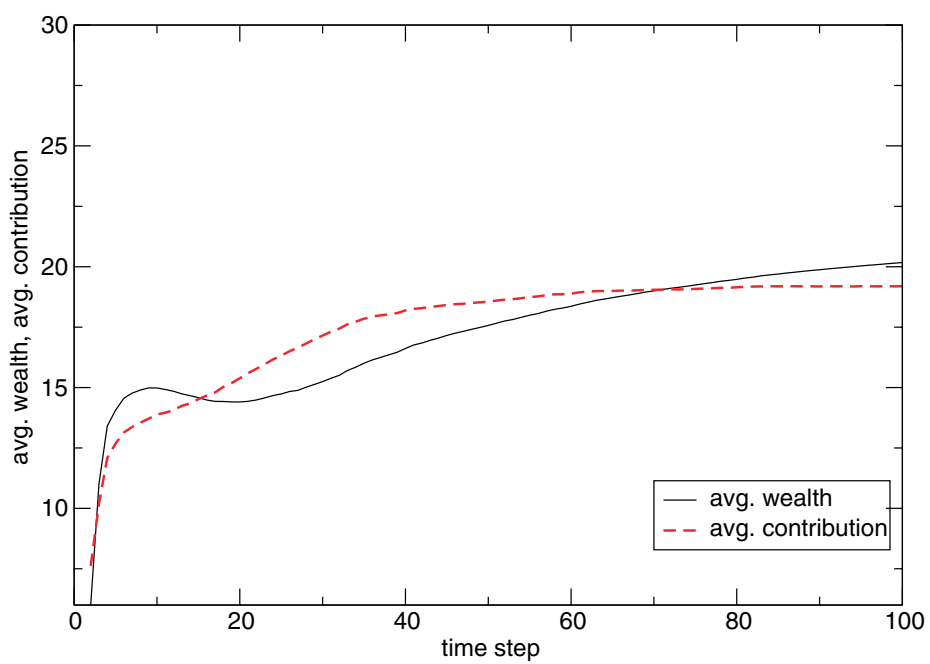

Fig. 2. Average wealth $\left(\left\langle\overline{w_{i}}\right\rangle\right)$ and contribution $\left(\left\langle q_{i}\right\rangle\right)$ as a function of time (steps); for medium rate of free-riders $\left(p_{R}=50 \%\right)$; with punishment plus bribery, and low rate of gossiping $\left(p_{g}=20 \%\right)$.

the wealth at the beginning. As soon as some free-riders are eliminated, the wealth increases again because the contribution increases.

When $p_{R}=50 \%$, the evolution of $\left\langle\overline{w_{i}}\right\rangle$ and $\left\langle q_{i}\right\rangle$ are depicted in Figs. $2-4$ for $p_{g}=20 \%, p_{g}=50 \%$, and $p_{g}=70 \%$ respectively. We see that the wealth no longer increases steadily (as in the previous cases). This is due to the fact that contribution does not stabilize as fast as in the other cases. The more gossiping, the more agents

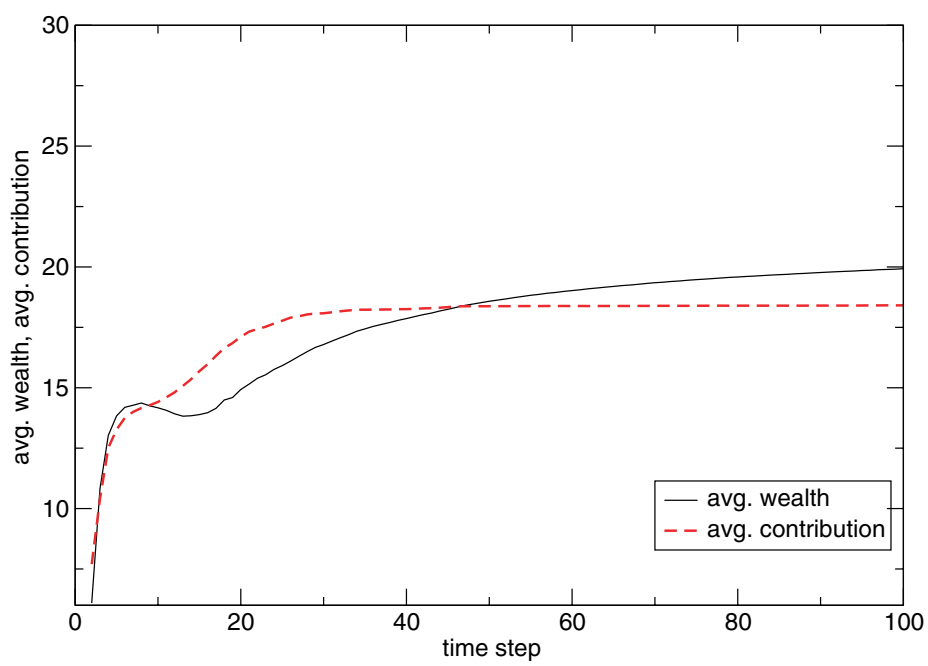

Fig. 3. Average wealth $\left(\left\langle\overline{w_{i}}\right\rangle\right)$ and contribution $\left(\left\langle q_{i}\right\rangle\right)$ as a function of time (steps); for medium rate of free-riders $\left(p_{R}=50 \%\right)$; with punishment plus bribery, and medium level of gossiping $\left(p_{g}=50 \%\right)$. 


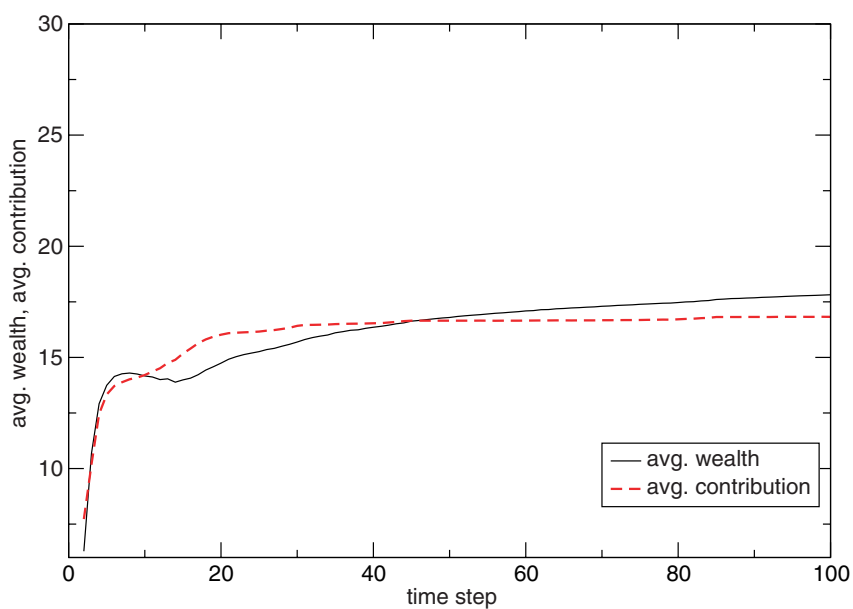

Fig. 4. Average wealth $\left(\left\langle\overline{w_{i}}\right\rangle\right)$ and contribution $\left(\left\langle q_{i}\right\rangle\right)$ as a function of time (steps); for medium rate of free-riders $\left(p_{R}=50 \%\right)$; with punishment plus bribery, and high level of gossiping $\left(p_{g}=\right.$ $70 \%)$.

are likely to change their blue and red beard lists. Also, due to bribes, information is not always the real one as the bribee will spread false information about the real beard color of the briber.

This phenomenon also occurs when $p_{R}$ is 25 or $75 \%$, but in those cases the prevailing factor is this percentage itself, not so much the other aspects.

Figure 5 summarizes these results. Regarding contribution, which, we remark, should be understood as the main measure, the worst situation happens when
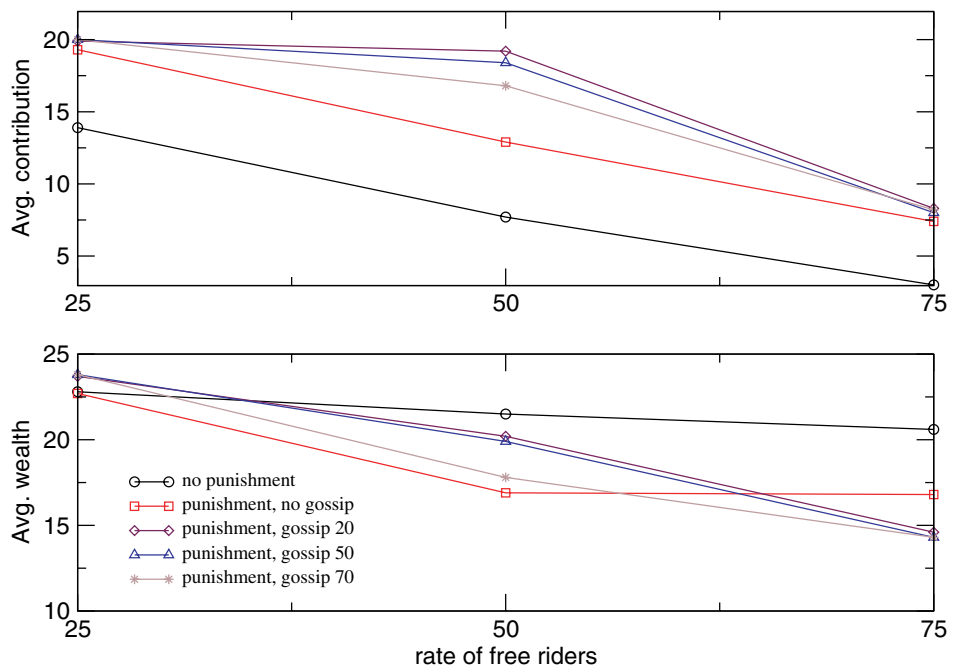

Fig. 5. Average contribution (top) and wealth (bottom) as function of rate of free-riders, for cases with and without punishment, and for low, medium, and high rate of gossiping. 
there is no punishment. Figures there agree with values given in Table 2. There is a significant increase in the contribution value when punishment and bribery is considered, as already known from the literature. Adding gossiping increases the contribution further, especially when the percentage of free-riders is equal that of high-contributors. In the cases where one or the other predominates, the increase is not significant.

Regarding wealth, comparing the four curves in Fig. 5 that are related to punishment and bribery, it is possible to draw two conclusions. First, gossiping does not pay off when there is a high rate of free-riders; see bottom of Fig. 5 for $p_{R}=75 \%$. Gossiping cannot compensate for the large presence of free-riders. For $p_{R}=25 \%$, the increase is modest: the reason is that there are too many subjects to be gossiped about. For $p_{R}=50 \%$, the increase is significant only when the rate of gossip is low to average.

Second, high gossip rate has a lower impact on the wealth (as compared to the case of contribution) because it causes a high level of punishment due to more information about who the free-riders are. When $p_{g}=70 \%$ for example, the wealth is at most equivalent to the case in which there is no gossip but just punishment. In the extreme case in which rates of gossiping and free-riders are high, the performance clearly degrades (though this happens for lower rates of gossiping as well, indicating that this performance is related to the high rate of free-riders, associated with information about their tags being spread out).

So far we have discussed the evolution of cooperation in macroscopic terms. We now report some prototypical behavior we have noticed in the simulation. One of the main advantages of an agent-based tool for simulation is exactly the possibility of following a microscopic behavior, even if it is then difficult to report individual behavior as the population of agents is big.

We discuss here two cases that may have more interest: the behavior and performance of an individual blue beard in an unfavorable setting $\left(p_{R}=75 \%\right)$, and two red beards in a setting with $p_{R}=50 \%$. In both cases, punishment, gossiping and bribery can be exercised.

The blue beard whose performance we follow is a greedy person: $\lambda_{i}=2.5$, meaning that she only accepts bribes that are at least 2.5 times higher than her own wealth. Of her four neighbors, two are blue beards and thus she contributes more than her minimum, at least in the beginning. This changes later when she is informed about other red beards. However, given that the rate of red beards is high, chances are that she will contribute towards $q_{\min _{H C}}$. In any case, she consistently has a bad performance because her contribution level is above the average in the population. This also causes her (and similarly other blue beards in this scenario) not to afford punishment, which improves red beards' performance.

A more interesting situation arises when we look at the two red beards in a setting with $p_{R}=50 \%$. The first one $\left(R_{1}\right)$ is not only a free rider; he is also not prone to spending his money bribing: $\beta_{1}=5 \%$. The second one $\left(R_{2}\right)$ is more 
generous: $\beta_{2}=48 \%$. In each round, $R_{1}$ receives a positive return of course but because news have spread that he has a red beard and because his neighbors in the grid are, unfortunately, very wealthy, these can afford to punish him and so $R_{1}$ is soon eliminated. $R_{2}$ however had more luck: one of its neighbors, a blue beard, is humble and does not demand a high bribe so $R_{2}$ is able to bribe her. He is even luckier: the other three neighbors were soon eliminated so that nobody else can observe his red beard. If there were no gossiping, $R_{2}$ would perform well and never be eliminated. However, those eliminated neighbors did, while still alive, spread the gossip about $R_{2}$ 's beard color so that eventually he was punished. The more red beards are eliminated, the more the remaining ones get punished. Eventually, $R_{2}$ is also eliminated. Red beards that are able to live long are either generous bribers or have neighbors that were eliminated very soon.

\section{Conclusion}

The idea of incorporating richer social mechanisms for agents' interactions such as having information about beard colors of other agents (here used as a metaphor for reputation), plus the possibility of punishment and bribery are interesting ones, as cooperation can then be tested in situations that are closer to the behavior of human beings that have social attachments.

Regarding the experimental methods and results, we remark the following. Having agents interact with their neighbors while also being eventually informed about reputation of distant agents in the grid modifies the dynamics of the game when compared to other settings of the public goods game that are discussed in the literature. Simulations performed under different conditions show qualitatively equal curves but that differ in the values for global average wealth and global contribution. There is a richer dynamics when the proportion of red and blue beards are nearly the same. It takes longer for the system to stabilize, but eventually it does.

One feature investigated in this paper is the evolution of cooperation in the presence of gossiping. As long as the number of free-riders is nearly the same as high-contributors, gossiping, i.e. spreading the news about others' reputation does affect agents contributions in a positive way, especially if this is combined with punishment and bribery (in the situation where there is no gossip, punishment or the absence of it does not change the dynamics qualitatively: contributions decrease with time).

For future work, we plan to extend this investigation in some ways: the main one is to explore is the study of the behavior of the system under different values for the cost of punishment. Although preliminary observation points to no qualitative difference, there might be some subtleties that our simulations were not able to show. Another interesting point would be to determine a phase diagram as a function of some key parameters to help define whether there are regions of prototypical behavior. 
Another step can be taken in the direction of having enforcement agents whose task would be to observe bribery, and then punish both bribee and briber so as to enforce social norms.

\section{Acknowledgments}

We would like to thank the anonymous reviewers and the editor for pointing out interesting research directions as well as taking their time to make comments to improve this research and make the results clearer. Both authors are partially supported by CNPq.

\section{References}

[1] Antal, T., Ohtsuki, H., Wakeley, J., Taylor, P. D. and Nowak, M. A., Evolution of cooperation by phenotypic similarity, Proc. Natl. Acad. Sci. USA (2009).

[2] Bergstrom, T., Evolution of social behavior: Individual and group selection, J. Econ. Perspect. 16(2) (2002) 67-78.

[3] Bergstrom, T. and Stark, O., How altruism can prevail in an evolutionary environment, Am. Econ. Rev. 83(2) (1993) 149-155.

[4] Bowles, S. and Gintis, H., The evolution of strong reciprocity: Cooperation in heterogeneous populations, Theor. Popul. Biol. 65(1) (2004) 17-28.

[5] Dawkins, R., The Selfish Gene (Oxford University Press, New York, 1976), 224 pp.

[6] de Jong, S. and Tuyls, K., Learning to cooperate in a continuous tragedy of the commons, in Proc. of the Int. Conf. on Autonomous Agents and Multiagent Systems (AAMAS), (2009), pp. 1185-1186.

[7] De Jong, S., Tuyls, K. and Verbeeck, K., Artificial agents learning human fairness, Padgham, L., Parkes, L., Müller, J. and Parsons, S. (eds.), in Proc. of the Yth International Joint Conference on Autonomous Agents and Multi-Agent Systems (AAMAS 2008), (2008), pp. 863-870.

[8] Eshel, I., Samuelson, L. and Shaked, A., Altruists, egoists, and hooligans in a local interaction model, Am. Econ. Rev. 88(1) (1998) 157-79.

[9] Gintis, H., Strong reciprocity and human sociality, J. Theor. Biol. 206(2) (2000) 169-179.

[10] Gintis, H., Smith, E. A. L. D. E. N. and Bowles, S., Costly signaling and cooperation, J. Theor. Biol. 213(1) (2001) 103-119.

[11] Gürek, Ö., Irlenbusch, B. and Rockenbach, B., The competitive advantage of sanctioning institutions, Science 312(5770) (2006) 108-111.

[12] Hamilton, W. D., The genetic evolution of social behavior ii, J. Theor. Biol. 7(1) (1964) 17-52.

[13] Henrich, J., Cooperation, punishment, and the evolution of human institutions, Science 312(5770) (2006) 60-61.

[14] Nowak, M. A., Five rules for the evolution of cooperation, Science 314(5805) (2006) $1560-1563$.

[15] Nowak, M. A. and May, R. M., Evolutionary games and spatial chaos, Nature 359 (1992) 826-829.

[16] Nowak, M. A., Tarnita, C. E. and Antal, T., Evolutionary dynamics in structured populations, Philos. Trans. R. Soc. B 365 (2010) 19-30.

[17] Santos, F. C., Santos, M. D. and Pacheco, J. M., Social diversity promotes the emergence of cooperation in public goods games, Nature 454(7201) (2008) 213-216. 
[18] Silva, R., Baraviera, A., Dahmen, S. R. and Bazzan, A. L. C., Dynamics of a public investment game: From nearest-neighbor lattices to small-world networks, C. Bruun (ed.), in Advances in Artificial Economics, The Economy as a Complex Dynamic System, No. 584, in Lecture Notes in Economics and Mathematical Systems (Springer, 2006), pp. 211-233.

[19] Silva, R., Bazzan, A. L. C., Baraviera, A. and Dahmen, S. R., Emerging collective behavior of financial dynamics in a public investment game, Physica A 371(2) (2006) 610-626. 